\title{
Neurohumoral Markers of Cardiac Autonomic Denervation After Surgical Ablation of Long- Standing Persistent Atrial Fibrillation
}

\author{
Alexey Evtushenko \\ Research Institute for Complex Issues of Cardiovascular Diseases \\ Vladimir Evtushenko \\ Cardiology Research Institute, Tomsk National Research Medical Centre \\ Anna Gusakova \\ Cardiology Research Institute, Tomsk National Research Medical Centre \\ Tatiana Suslova \\ Cardiology Research Institute, Tomsk National Research Medical Centre

\section{Yulia Varlamova} \\ Cardiology Research Institute, Tomsk National Research Medical Centre \\ Konstantin Zavadovskiy \\ Cardiology Research Institute, Tomsk National Research Medical Centre

\section{Denis Lebedev} \\ Research Institute for Complex Issues of Cardiovascular Diseases \\ Elena Pavlyukova \\ Cardiology Research Institute, Tomsk National Research Medical Centre \\ Sergey Mamchur ( $\nabla$ sergei_mamchur@mail.ru ) \\ Research Institute for Complex Issues of Cardiovascular Diseases
}

\section{Research Article}

Keywords: long-standing persistent atrial fibrillation, autonomic nervous system, norepinephrine, efficacy of radiofrequency ablation.

Posted Date: September 24th, 2021

DOI: https://doi.org/10.21203/rs.3.rs-860380/v1

License: (c) (1) This work is licensed under a Creative Commons Attribution 4.0 International License. Read Full License 


\section{Abstract}

Background. The autonomic nervous system (ANS) plays an important role in modulation of cardiac electrophysiology and arrhythmogenesis. Disorders of the ANS components can be a prognostic factor of an unfavorable course of cardiovascular diseases. The aim of the study was to evaluate the association between efficacy of radiofrequency (RF) surgical ablation of long-standing persistent atrial fibrillation (AF) and neurohumoral transmitter levels as well as correlation between these data with myocardial iodine-123-metaiodobenzylguanidine (123/-MIBG) uptake.

Methods. Two groups of patients with acquired valvular heart disease were compared: patients with surgical AF ablation and patients with sinus rhythm.

Results. The decrease of norepinephrine (NE) level in coronary sinus has a direct association with the heart-to-mediastinum ratio $(p=0.02)$ and negative correlation with ${ }^{123}$ I-MIBG uptake defect $(p=0.01)$. NE level decreased significantly after the main stage of surgery, both in patients with $A F(p=0.0098)$ and sinus rhythm $(p=0.0039)$. Furthermore, the significance $(p=0.001)$ of the aortic root and coronary sinus gradient of $\mathrm{NE}$ level $0.405 \mathrm{pg} / \mathrm{mL}$ was determined as a cut-off value for efficacy evaluation of the RF denervation.

Conclusion. The practical significance of the obtained results lies in the possibility of using the technique to predict the efficacy of the 'Maze-IV' procedure, which will allow assessing the risk of AF recurrence after ablation.

\section{Introduction}

The autonomic nervous system (ANS) plays an important role in modulation of cardiac electrophysiology and arrhythmogenesis [3]. Disorders of the ANS components can be a prognostic factor of an unfavorable course of cardiovascular diseases.

Many studies have contributed to a better understanding of the anatomy and physiology of the ANS and have provided evidence of the relationship between ANS and clinically significant arrhythmias [3]. They demonstrated the prospects for the development of this area of research in arrhythmology.

Neurohumoral markers are an important discovery that reflect the autonomic activity of the heart. Currently, there is no universal method for studying the ANS, and modern research methods impose certain requirements that are not always possible to fulfill. Therefore, the identification of specific markers and triggers of ANS in the development of arrhythmias can become an alternative to many invasive methods in studying autonomic cardiac activity. This is especially relevant for patients with long-standing persistent atrial fibrillation (AF).

$\mathrm{AF}$ is one of the most common complications of cardiac surgery. It occurs: in $30 \%$ of cases after coronary artery bypass surgery, in $40 \%$ of cases after valvular correction and in $50 \%$ of cases after a combination of these interventions [1]. The risk of postoperative AF recurrence will reach $100 \%$ if there is $\mathrm{AF}$ in the previous medical history [12]. Even without interventions, cardiovascular surgery patients have a high probability of postoperative AF occurrence. Patients with insufficient RF ablation of AF have a high risk of recurrence in the postoperative period. It is necessary to have clear criteria for $A F$ treatment endpoint to prevent recurrence. In terms of practical significance, the evaluation of the ANS modulation of the myocardium after surgical treatment can serve as a predictor of AF recurrence, as well as other undesirable cardiac events.

The aim of this study was to identify criteria for autonomic heart modulation after surgical treatment of long-standing persistent AF concomitant with valvular heart disease correction.

\section{Methods}

\section{Patients characteristics}

A randomized, prospective study was performed. The object of the study were patients with mitral valve disease recommended for surgical correction. 68 subjects were included in the study; 53 of them had a mitral valve disease complicated with long-standing persistent AF, 15 patients had sinus rhythm (SR) (Table 1). 
Table 1

- Main preoperative clinical and instrumental parameters of patients with AF (group I) and SR (control group II), who underwent sympathetic nervous system examination $(n=68)$

\begin{tabular}{|c|c|c|c|}
\hline \multirow[t]{2}{*}{ Parameter } & Group I. AF: AHD treatment + RFA $(n=53)$ & Goup II. Control group with SR $(n=15)$ & \multirow[t]{2}{*}{ p-value } \\
\hline & $\mathrm{Me}(\mathrm{Q} 1 ; \mathrm{Q} 3)$ & $\mathrm{Me}(\mathrm{Q} 1 ; \mathrm{Q} 3)$ & \\
\hline Age (years) & $59.5(53 ; 64)$ & $57.0(53 ; 64)$ & 0.9 \\
\hline LA diameter (mm) & $52,0(47 ; 55)$ & $46,0(40 ; 46)$ & 0.002 \\
\hline RV diameter (mm) & $24.0(21 ; 28)$ & $22.0(22 ; 24)$ & 0.3 \\
\hline IVS (mm) & $10.0(9 ; 10)$ & $9.0(9 ; 10)$ & 0.7 \\
\hline $\operatorname{LVEDD}(\mathrm{mm})$ & $53.75(51 ; 58)$ & $53.0(47 ; 56)$ & 0.5 \\
\hline LVESD (mm) & $33.5(32 ; 39)$ & $32.5(29 ; 34)$ & 0.2 \\
\hline LVEF (Teicholz) (\%) & $61.0(56 ; 70)$ & $69.0(66 ; 75)$ & 0.2 \\
\hline LVEF (Simpson biplane) (\%) & $66.0(60 ; 68)$ & $66.0(65 ; 72)$ & 0.3 \\
\hline LVEDV (mL) & $132.5(106 ; 194)$ & $112.0(104 ; 142)$ & 0.6 \\
\hline LVESV (MI) & $45.5(35 ; 71)$ & $49.0(31 ; 50)$ & 0.5 \\
\hline RVSP (mmHg) & $44.0(40 ; 50)$ & $40.0(38 ; 61)$ & 0.9 \\
\hline EuroSCORE, \% & $3.07(1.96 ; 4.68)$ & $2.27(2.08 ; 4.81)$ & 0.9 \\
\hline EuroSCORE, points & $4.0(3 ; 6)$ & $3.0(3 ; 5)$ & 0.6 \\
\hline 6-minute walking test $(\mathrm{m})$ & $220.0(200 ; 256)$ & $322.0(250 ; 356)$ & 0.02 \\
\hline
\end{tabular}

Comments. LA - left atrium; RV - right ventricle; IVS - interventricular septum; LVEDD - left ventricular end-diastolic diameter; LVESD - left ventricular endsystolic diameter; LVEF - left ventricular ejection fraction; LVEDV - left ventricular end-diastolic volume; LVESV - left ventricular end-systolic volume; RVSP - right ventricular systolic pressure.

The including criterion for the study was the preserved function of the sinus node (SN), which had been determined by intraoperative electrophysiological (EP) study (Fig. 1). Seven days before the surgery patients were taken off medications that affect the rhythm and conduction of the heart. Further, the intraoperative EP study with the assessment of sinus node recovery time (SNRT), corrected sinus node recovery time (CSNRT) and Wenckebach point was performed. Patients with preserved SN function were included in the study and randomized into 2 groups using envelopes. In the first group RF ablation was combined with postganglionic plexi lesions in accordance with the N. Doll (2008) scheme, in the second group, lesions were performed in accordance with the 'Maze-IV' scheme without additional ablation of postganglionic plexi.

With normal SN function, RF ablation was performed according to the 'Maze IV' scheme (using penetrating technique) with both atrial appendages sealing and additional RF exposure to the postganglionic plexi. The control group $(n=15)$ included patients with SR who underwent surgery only due to valvular lesions.

Additional criteria for exclusion from the study: comorbidity, multivessel coronary disease, pericardial adhesions, multiple organ failure and the patient srefusal $\rightarrow$ participate $\in$ thestudy. Theprimaryendp $\oint$ swerecardiovascarm or tality and maj or cardiovascarevents, suchasmyocardial $\in$ $s$ refusal to continue participation in the study.

Each patient underwent a standard set of clinical and laboratory tests, including blood and urine tests, X-ray examination, echocardiography, carotid artery sonography, spirometry, coronary angiography (in accordance with the recommendations [2]). Planar scintigraphy with ${ }^{123} \mathrm{I}$-metaiodbenzylguanidine $\left({ }^{123} \mathrm{I}\right.$ MIBG) was performed before and after the surgery. ${ }^{123}$-MIBG is an analogue of guanethidine adrenergic blocker allowing visualization of sympathetic innervation of the heart with the assessment of myocardial adrenergic nerve activity in various cardiac pathologies, including arrhythmias [16]. The following parameters were assessed during myocardial scintigraphy: heart-to-mediastinum ratio, early and late radiopharmaceutical (RP) washout, RP uptake defect.

A 6-minute walking test was used to determine the functional class of heart failure.

Patients with persistent AF (group I) were statistically significantly different from patients with SR (group II) in the left atrial (LA) diameter and in the results of the 6-minute walking test, which is most likely due to AF.

Chronic rheumatic heart disease was diagnosed in patients of group I in $50 \%$ of cases and in group II in $44.4 \%$ of cases. Connective tissue dysplasia was found in $28 \%$ and $44.4 \%$ of cases, respectively. Some of patients from both groups required coronary artery bypass grafting (1-2 grafts).

Surgical treatment was performed in accordance with the recommendations for the management of patients with valvular heart diseases (ESC/EACTS, 2017). The frequency of mitral valve (MV) repair with annuloplasty rings in the groups was $50 \%$ and $55.5 \%$, respectively, and MV prosthetic valve replacement was performed in $33.3 \%$ of cases in both groups. A number of patients required multi-valvular interventions: single-stage tricuspid valve repair was performed in both groups in $28 \%$ and $22.2 \%$ of cases, respectively. Some $(n=4)$ patients underwent simultaneous intervention on the aortic valve.

Antiarrhythmic therapy in group I was prescribed in case of paroxysmal AF.

Page $3 / 12$ 


\section{Neurotransmitter levels assessment}

Blood samples were collected intraoperatively from the ascending aorta (Ao) and coronary sinus (CS) before and after the main stage of the surgery. The study was performed in the absence of any medications that could affect the catecholamine levels in the samples (in case of catecholamine injection, patients were excluded from the study). NE level in blood were assessed before and after RF ablation. Plasma catecholamine level was determined by enzyme immunoassay. The 'Noradrenalin ELISA kit' (IBL, Hamburg, Germany) was used for quantitative determination of plasma NE level.

\section{Statistical processing of the obtained data}

The data obtained are described using the Median, upper an lower quartiles (Me, Uq, Lq). The significance of intergroup differences was assessed using the Student T-Test and the Mann-Whitney U-test. To assess the significance of intragroup differences (dynamic of indicators before/after exposure within one group), the Wilcoxon test was used. P-value was 0.05 , the confidence interval $R_{\text {conf }}=0.95$. Discriminant analysis was used to build a model for the assessment of the RF ablation efficacy based on neurohumoral factors.

\section{Results}

In patients with long-standing persistent AF, the median heart-to-mediastinum ratio in the early and late phase of the study before the intervention did not differ significantly from the group of patients with SR $(1.75(1.59 ; 1.91)$ and $2.06(1.7 ; 2.1)$ respectively, $p=0.1)$. The washout rate also did not significantly differ between the groups $(24.5(10.8 ; 41.1) \%$ and $24.3(12.3 ; 29.9) \%$, respectively, $p=0.15)$. The ${ }^{123 I}$-MIBG uptake defect before surgery in both groups was comparable and did not differ significantly $(13.0(7.0 ; 24.0) \%$ and $19.0(9.5 ; 23.5 \%) \%$, respectively, $p=0.59)$.

Based on the preoperative data, there were no statistically significant differences in sympathetic nervous system tonus in both groups.

Patients underwent a postoperative examination 4 week after surgery, which included several studies mentioned above (Table 2).

Table 2

Main postoperative clinical and instrumental parameters of patients with AF (group I) and SR (control group II), who underwent sympathetic nervous system examination $(\mathrm{n}=68)$

\begin{tabular}{|c|c|c|c|}
\hline \multirow[t]{2}{*}{ Parameter } & \multirow{2}{*}{$\begin{array}{l}\text { Goup I. AF: AHD treatment + RFA }(n=53) \\
\text { Me (Q1; Q3) }\end{array}$} & \multirow{2}{*}{$\begin{array}{l}\text { Goup II. Control group with SR }(n=15) \\
\text { Me (Q1; Q3) }\end{array}$} & \multirow[t]{2}{*}{ p-value } \\
\hline & & & \\
\hline LA diameter before the surgery (mm) & $45.0(44 ; 49)$ & $39.0(25 ; 41)$ & 0.005 \\
\hline RV diameter before the surgery ( $\mathrm{mm})$ & $23.5(20 ; 27)$ & $20.0(20 ; 22)$ & 0.13 \\
\hline IVS before the surgery (mm) & $10.0(9 ; 10)$ & $9.0(9 ; 10)$ & 0.5 \\
\hline LVEDD before the surgery (mm) & $49.0(47 ; 54)$ & $46.0(44 ; 48)$ & 0.1 \\
\hline LVESD before the surgery (mm) & $33(30 ; 37)$ & $30.0(26 ; 35)$ & 0.2 \\
\hline LVEDV before the surgery $(\mathrm{mL})$ & $119.5(90 ; 127)$ & $87.0(72 ; 98)$ & 0.2 \\
\hline LVESV before the surgery (Ml) & $40.5(34 ; 53)$ & $31.0(27 ; 37)$ & 0.03 \\
\hline LVEF (Teicholz) before the surgery (\%) & $61.5(57 ; 65)$ & $64.0(61 ; 75)$ & 0.04 \\
\hline LVEF (Simpson biplane) before the surgery (\%) & $60.5(58 ; 66)$ & $67.0(64 ; 70)$ & 0.08 \\
\hline RVSP before the surgery (mmHg) & $35.0(30 ; 40)$ & $35.0(28 ; 37)$ & 0.5 \\
\hline CPB time (min) & $120.0(110 ; 161)$ & $120.0(113 ; 140)$ & 0.6 \\
\hline ACC time (min) & $70.0(57 ; 98)$ & $92.0(79 ; 110)$ & 0.3 \\
\hline 6-minute walking test after the surgery $(\mathrm{m})$ & $406.5(380 ; 435.5)$ & $412.0(290 ; 450)$ & 0.7 \\
\hline
\end{tabular}

The efficacy of AF RF ablation was evaluated using postoperative ${ }^{123}$ I-MIBG scintigraphy and the neurotransmitters (metanephrine, normetanephrine, NE) levels assessment in the blood from Ao and CS were assessed.

In patients with atrial RF fragmentation (group I), the heart-to-mediastinum ratio after intervention in the late phase of the study was significantly lower compared to the group of patients with SR: $(1.5(1.4 ; 1.6)$ and $1.8(1.56 ; 1.83)$, respectively, $\mathrm{p}=0.02)$. In group I, the uptake defect was significantly higher than in control group II $(25.0(24.0 ; 35.0) \%$ and $15.0(12.0 ; 20.0) \%$, respectively, $p=0.01)$.

A statistically significant $(p=0.0098)$ decrease in the NE level in patients with RF ablation was found in the CS blood collected after the main stage of surgery compared to preoperative level. In the control group, there was no significant difference $(p=0.2)$ in the NE level in the CS blood.

There was a statistically significant intergroup difference $(p=0.0039)$ in the postoperative NE level (Fig. 2$)$. This correlates with scintigraphy data: 1$)$ the decrease in the heart-to-mediastinum ratio and reduction of the CS NE level in patients with RF ablation (group I) compared to group II after the intervention - 
a direct correlation, 2) the increased RP uptake defect and reduced CS NE level - an inverse relationship. Meanwhile, the differences between the subgroups with the 'Maze-IV' procedure in the heart-to-mediastinum ratio (Fig. 3), the RP washout rate (Fig. 4) and the RF uptake defect (Fig. 5) were statistically insignificant.

Discriminant analysis was used to build a model for the assessment of the RF ablation efficacy based on neurohumoral factors. The Wilks` Lambda criterion was $p=0.0037$, indicating the adequacy of the created model (Table 3 ).

Table 3

Assessment of the adequacy of the RF denervation efficacy model based on the Wilks' Lambda criterion

\begin{tabular}{|c|c|c|c|c|c|c|}
\hline \multirow[t]{2}{*}{ Parameter } & \multicolumn{6}{|c|}{$\begin{array}{l}\text { Wilks' Lambda: } 0.56315 \text { approx. F }(3.23)=5.9471 \text {. } \\
p<0.0037\end{array}$} \\
\hline & Wilks`'Lambda & Partial Lambda & F-remove (1.23) & p-value & Toler. & 1-Toler. (R-Sqr.) \\
\hline NE level gradient $(\mathrm{Ao} / \mathrm{CS})(\mathrm{pg} / \mathrm{mL})$ & 0.89 & 0.64 & 13.2 & 0.001 & 0.99 & 0.01 \\
\hline Normetanephrine level gradient (Ao/CS) $(\mathrm{pg} / \mathrm{mL})$ & 0.59 & 0.94 & 1.41 & 0.24 & 0.99 & 0.006 \\
\hline
\end{tabular}

The difference in the NE levels after the main stage of the surgery is a significant factor. This parameter was calculated separately for each patient as follows:

$\Delta N A=N A_{a a}-N_{c s}$,

where NA - noradrenaline; aa - ascending aorta; cs - coronary sinus.

Distribution in groups was carried out based on linear classification. The total number of correct relationships to a group was $89 \%$, that indicates a high sensitivity and specificity of the created model (Table 4).

Table 4

Classification matrix

\begin{tabular}{|llll|}
\hline Group & The percentage of correct assignments & G_1:1 & G_2:2 \\
& & $\mathbf{p}=\mathbf{0 . 7 0 3 7}$ & $\mathbf{p}=\mathbf{0 . 2 9 6 3}$ \\
\hline G_1:1 & 94.7 & 50 & 3 \\
\hline G_2:2 & 75.0 & 3 & 12 \\
\hline Total & 88.9 & 53 & 15 \\
\hline Based on the coefficients for the canonical linear discriminant function (CLDF), the following equation was calculated: \\
\hline
\end{tabular}

$\mathrm{CLDF}=0.77+0.003 \Delta \mathrm{NA}_{\mathrm{after}}$

The values of the centroid function were obtained (Fig. 6) by substituting the average values of predictors in the group of patients with postganglionic nerve plexi ablation (control group) into the discriminant equation.

Thus, the centroid was 0.55 in patients with ablated postganglionic plexi, and minus 1.36 in the group without ablation. The cut off value $\Delta H A=-0.405$ $\mathrm{pg} / \mathrm{mL}$. The efficacy of cardiac denervation can be calculated using the CLDF equation and the ratio of the obtained data to the centroids for each group and the group separation boundary (Fig. 6).

\section{Discussion}

$\mathrm{AF}$ is a widespread heart rhythm disorder. AF is the result of interaction between the trigger that initiates arrhythmia and the substrate that keeps it persistent. Despite a large number of studies, mechanisms of AF initiation are not fully understood [15]. However, it is generally accepted that sympathovagal imbalance can lead to paroxysmal supraventricular arrhythmia [15] and paroxysmal AF [4]. Therefore, local denervation of the heart can play a major role in the surgical treatment of long-standing persistent AF.

\section{Influence of sympathetic and parasympathetic nervous activity on cardiac electrophysiology}

The effect of the ANS sympathetic component on the EP of the heart is complex. It depends on the state of the myocardium itself (the presence of a substrate for AF). The predominance of sympathetic activity in an intact heart leads to a shortening of the cardiac action potential (AP) duration and to decrease of the repolarization dispersion. On the contrary, when the structure of the myocardium damages, sympathetic activity leads to an increase in the repolarization dispersion and to the early afterdepolarization generation [3], which provides proarrhythmogenic effect. However, not only the ANS sympathetic component can lead to arrhythmias. A few studies (Patterson et al.) based on the analysis of heart rate variability have shown that AF paroxysm can be the result of combined activation of the sympathetic and parasympathetic components [5-8]. Sympathetic activity is the cause of increased calcium transit. The increase of the parasympathetic tonus leads to the atrial effective refractory period shortening. The difference between the calcium transit and the AP duration

Page 5/12 
(normally interdependent) leads to an increase of the $\mathrm{Na}+\mathrm{Ca}++$ pump activity, which is responsible for the generation of early afterdepolarization and trigger activity $[3,5]$.

There are two levels of vegetative regulation of heart activity - extracardiac (central) and intracardiac (internal). Activation of central regulatory mechanisms does not always lead to an imbalance in the postganglionic neurons of the heart. Studies by Choi et al. have shown that external and internal mechanisms of activation of sympathetic heart tonus are related. Some unexpected results show that although activation of extracardiac ANS contributed to the onset of most AF paroxysms, in some cases ( $11 \%$ of AF paroxysms in the study by Choi et al.), internal nervous activity of the heart occurred before the external one and led to the development of AF paroxysms [4]. Moreover, the internal sympathetic activity of the heart can activate the external parts of the ANS, leading to the negative consequences. This mechanism is the basis of AF and it is implemented by sympathovagal imbalance [4].

Many qualitative and quantitative methods have been proposed for the study of the ANS. One of the most common is the analysis of heart rate variability. However, this method has at least two limitations. First, the method is functional - it reflects only the sympathovagal balance between the ANS components, not their absolute activity. Second, this analysis requires an intact SN that mediates an adequate cardiac response to ANS activity. Some patients with longstanding persistent AF and heart failure have comorbid SN dysfunction. This imposes restrictions in the use of functional methods for studying the ANS tonus. Therefore, these methods are not suitable for this group of patients [3]. To assess the sympathetic tonus in such patients, quantitative methods of ANS studying are used. The most common of them is ${ }^{123}$-MIBG scintigraphy. However, this method has an important drawback - the lack of clear diagnostic criteria [9]. Other methods are not specific enough to determine cardiac autonomous activity or they are quite time-consuming, which imposes restrictions on their use. Thus, there is no universal method for determining the ANS activity of the heart [10].

As mentioned above, the determination of neurohumoral factors levels for the myocardial vegetative activity studying can be an alternative to the aforementioned methods. Determination of such mediators as metanephrine, normetanephrine and NE in the Ao and CS blood can provide an assessment of the local vegetative activity of the heart.

This study shows that neurohumoral markers can serve as a quantitative criterion for the RF ablation efficacy in long-standing persistent AF. Among the markers of vegetative activity of the heart - NE plays a special role.

The main principle of surgical treatment of AF is the ablation of all abnormal pathways in order to ensure the normal conduction from the SN to the underlying parts of the cardiac conduction system. In addition to interrupting re-entry circles and isolating trigger zones, sympathetic plexi are similarly ablated during the procedure, which affects the activity of the sympathetic ANS component, including the NE level as a neurohumoral marker of vegetative activity of the heart.

The difference of NE level in Ao and CS blood after the main stage of surgery is an important factor in the RF ablation efficacy assessment. During evaluation of the quality model of atrial RF denervation based on the Wilks ' Lambda criterion showed that the criterion itself was 0.89 , and the ' $p$ ' for the postoperative 'Ao/CS' NE gradient level was 0.001 , which indicates a significant difference in the NE levels after the ablation. These data are correlated with scintigraphy ones: a decrease in the heart-to-mediastinum ratio and an increase in the RF uptake defect are correlated with a decrease in the CS blood NE level.

Additionally, a comparative analysis of the 'Maze-IV' procedure was performed with and without additional ablation of sympathetic postganglionic plexi. The data obtained shown that the full set of the 'Maze-IV' lesions allows performing heart denervation without additional impact on the sympathetic plexi.

It is necessary to consider the relatively small number of patients included in the study. Although the results of the study shown the importance of determining the NE level in the assessment of autonomic atrial denervation, further studies is necessary to obtain more data.

\section{Conclusion}

1. Neurohumoral markers of the autonomical nervous system in the postoperative period correlate with the presence/absence of local autonomic denervation during radiofreequency ablation. 2 . The gradient of the norepinephrine level in the aorta and coronary sinus is a marker of sympathetic denervation of the heart. 3. The cut off value between high-quality and low-quality denervation is $-0.405 \mathrm{pg} / \mathrm{mL}$. 4 . The practical significance of these results is the possibility of using the technique to predict the efficacy of the 'Maze-IV' procedure and assess of the atrial fibrillation recurrence risk. 5 . The method of assessment is more accurate, as it is quantitative and imposes fewer requirements for a patient with cardiovascular disease.

\section{Abbreviations}

${ }^{123}$ I-MIBG - 123I-metaiodbenzylguanidine; ACC - aortic cross-clamp; AF - atrial fibrillation; AHD - acquired heart diseases; ANS - autonomic nervous system; Ao - aorta; CLDF - canonical linear discriminant function; CPB - cardiopulmonary bypass; CS - coronary sinus; CSNRT - corrected sinus node recovery time; EP - electrophysiology; IVS - interventricular septum; LA - left atrium; LVEDD - left ventricular end-diastolic diameter; LVEDV - left ventricular end-diastolic volume; LVEF - left ventricular ejection fraction; LVESD - left ventricular end-systolic diameter; LVESV - left ventricular end-systolic volume; MV - mitral valve; NE - norepinephrine; RP - radiopharmaceutical; RFA - radiofrequency ablation; RV - right ventricle; RVSP - right ventricular systolic pressure; SN - sinus node; SND - sinus node dysfunction; SNRT - sinus node recovery time; SR - sinus rhythm.

\section{Declarations}

Ethics Approval and consent to participate 
The study was conducted according to the guidelines of the Declaration of Helsinki, and approved by the Institutional Ethics Committee of Research Institute for Complex Issues of Cardiovascular Diseases and Cardiology Research Institute, Tomsk National Research Medical Centre, Russian Academy of Sciences. Informed consent was obtained from all subjects involved in the study.

\section{Consent for publication}

Not applicable.

\section{Availability of Data and Materials}

The datasets used and analyzed during the current study are available from the corresponding author upon reasonable request.

\section{Competing interests}

The authors declare that they have no competing interests.

\section{Funding}

This research received no external funding.

\section{Author's Contributions}

A.E. - conceptualization; V.E. - performing the surgery and taking specimens of blood; A.G., T.S., Yu.V. and K.Z. - performing the SPECT studies; D.L. - writing the draft version of the manuscript; S.M. - writing (review and editing). All authors have read and agreed to the published version of the manuscript.

\section{Acknowledgments}

None.

\section{References}

1. Lomivorotov V.V., Efremov S.M., Pokushalov E.A., Boboshko V.A. Atrial fibrillation following cardiac surgery: pathophysiology and prevention techniques (part 2). Messenger of Anesthesiology And Resuscitation. 2017;14(2):64-71. (In Russ.) https://doi.org/10.21292/2078-5658-2017-14-2-64-71.

2. Hindricks G., Potpara T., Dagres N. et al. 2020 ESC Guidelines for the diagnosis and management of atrial fibrillation developed in collaboration with the European Association for Cardio-Thoracic Surgery (EACTS): The Task Force for the diagnosis and management of atrial fibrillation of the European Society of Cardiology (ESC) Developed with the special contribution of the European Heart Rhythm Association (EHRA) of the ESC // European Heart Journal 2021; 42(5):373-498.

3. Shen, Mark J. and Douglas P. Zipes. Role of the autonomic nervous system in modulating cardiac arrhythmias. Circulation research 2014;114(6):100421.

4. Choi, E., Shen, M.J., Han, S., et al. Intrinsic cardiac nerve activity and paroxysmal atrial tachyarrhythmia in ambulatory dogs. Circulation 2010;121(24):2615-23.

5. Chen PS, Tan AY. Autonomic nerve activity and atrial fibrillation. Heart Rhythm. 2007;4(3 Suppl):S61-S64. doi:10.1016/j.hrthm.2006.12.006.

6. Tomita T, Takei M, Saikawa Y. et al. Role of autonomic tonus in the initiation and termination of paroxysmal atrial fibrillation in patients without structural heart disease. J Cardiovasc Electrophysiol. 2003;14:559-564.

7. Patterson E, Lazzara R, Szabo B, Liu H, Tang D, Li YH, Scherlag BJ, Po SS. Sodium-calcium exchange initiated by the Ca2 + transient: an arrhythmia trigger within pulmonary veins. J Am Coll Cardiol. 2006;47: 1196-1206.

8. Patterson E, Jackman WM, Beckman KJ, Lazzara R, Lockwood D, Scherlag BJ, Wu R, Po S. Spontaneous pulmonary vein firing in man: relationship to tachycardia-pause early afterdepolarizations and triggered arrhythmia in canine pulmonary veins in vitro. J Cardiovasc Electrophysiol. 2007;18:10671075.

9. Cygankiewicz I, Zareba W. Heart rate variability. Handb Clin Neurol. 2013; 117:379-93.

10. Grassi G, Esler M. How to assess sympathetic activity in humans. J Hypertens. 1999; 17: 719-34

11. Evtushenko V.V., Makogonchuk I.S., Evtushenko A.V. Rules and principles of patient selection for surgical treatment of acquired heart diseases complicated by atrial fibrillation. The Siberian Journal of Clinical and Experimental Medicine. 2017;32(3):29-34. (In Russ.)

https://doi.org/10.29001/2073-8552-2017-32-3-29-34.

12. Bogachev-Prokofyev A.V., Pivkin A.N., Syrtseva Y.V., Zheleznev S.I., Afanasyev A.V., Sharifulin R.M., Karaskov A.M. Combinesd treatment of paroxysmal atrial fibrillation in patients with mitral valve disease: analysis of long-term outcomes. Journal of Arrhythmology. 2017;(87):16-22. (In Russian).

13. J.M. Cao, M.C. Fishbein, J.B. Han, et al., Relationship between regional cardiac hyperinnervation and ventricular arrhythmia, Circulation 2000;101:19601969.

14. J.C. Hardwick, S.E. Ryan, E. Beaumont, et al., Dynamic remodeling of the Guinea pig intrinsic cardiac plexus induced by chronic myocardial infarction, Auton. Neurosci. 181 (2014) 4-12.

15. Chen PS, Chen LS, Fishbein MC, Lin SF, Nattel S. Role of the autonomic nervous system in atrial fibrillation: pathophysiology and therapy. Circ Res. 2014;114(9):1500-1515. doi:10.1161/CIRCRESAHA.114.303772.

Page $7 / 12$ 
16. lodine-123 metaiodobenzylguanidine images reflect intense myocardial adrenergic nervous activity in congestive heart failure independent of underlying cause/Imamura, Yoshihiro [et al.] //Journal of the American College of Cardiology 1995;26(7):1594-1599.

\section{Figures}

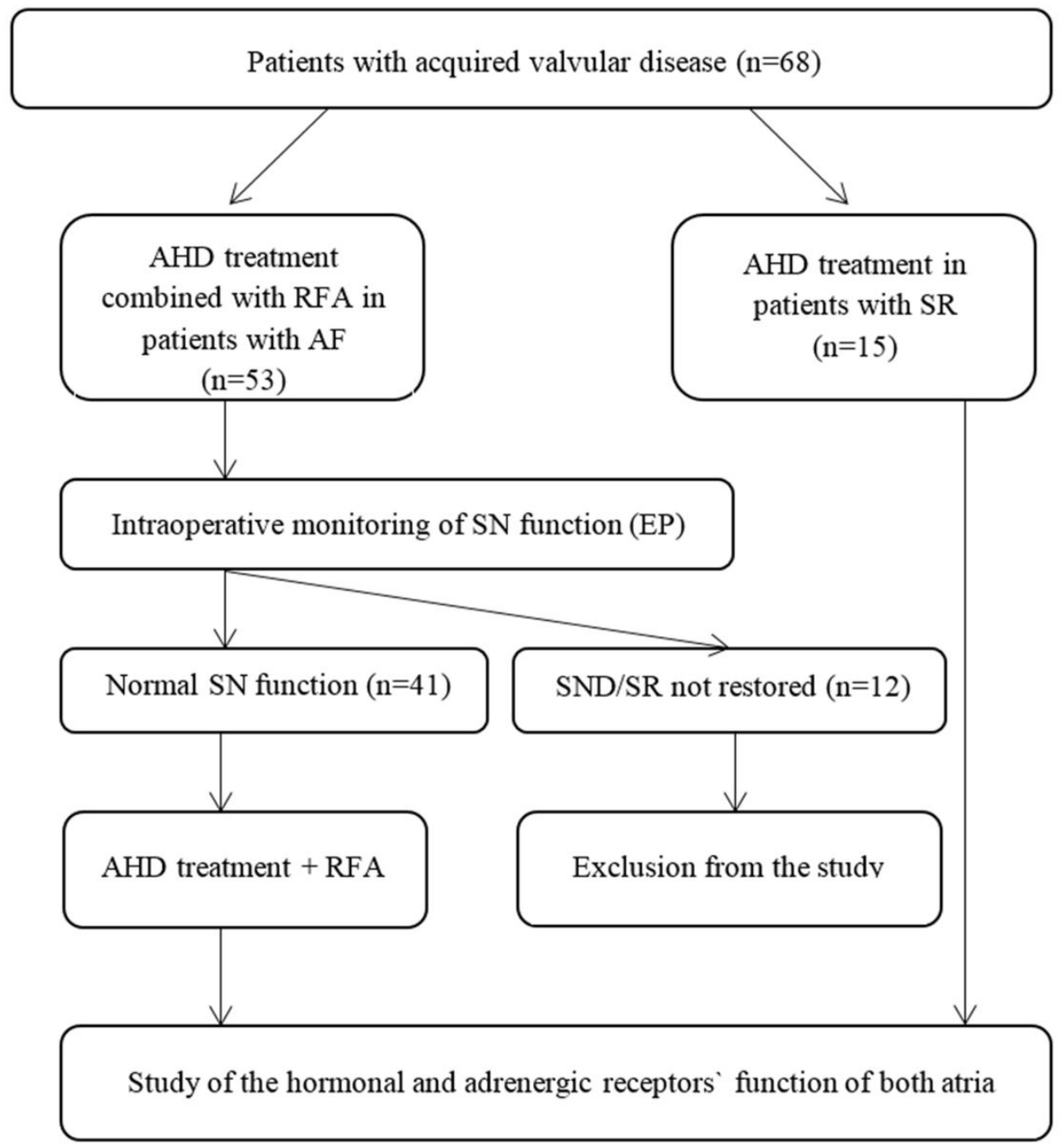

Figure 1

The design of the study. Note: AHD - acquired heart diseases; RFA - radiofrequency ablation; SR - sinus rhythm; SN - sinus node; EP - electrophysiological study; SND - sinus node dysfunction 
Boxplot by Group

Norepinephrine $(\mathrm{pg} / \mathrm{mL})$ in coronary sinus after surgery: $F(1 ; 33)=8.0488 ; p=0.0077$; $\mathrm{KW}-\mathrm{H}(1 ; 35)=7.84 ; \mathrm{p}=0.0051$

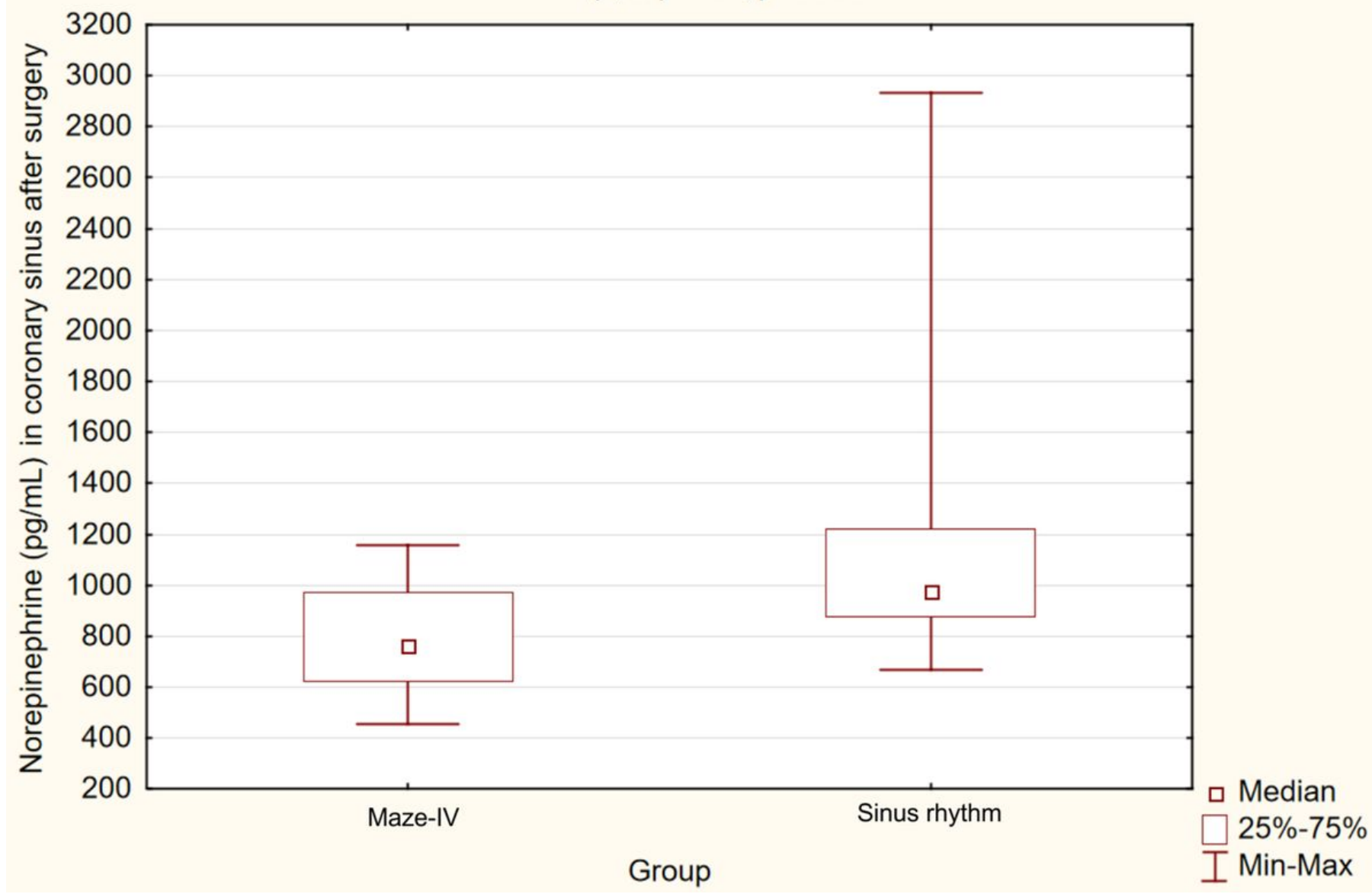

Figure 2

$\mathrm{NE}$ level in $\mathrm{CS}(\mathrm{pg} / \mathrm{mL})$ after the main stage of surgery 
Heart-to-mediastinum ratio in the late phase of the study

$H / M d 1: \quad F(2 ; 51)=4,8495 ; p=0,0118$;

$\mathrm{KW}-\mathrm{H}(2 ; 54)=8,6316 ; \mathrm{p}=0,0134$

H/Md2: $F(2 ; 53)=8,1893 ; p=0,0008$;

$\mathrm{KW}-\mathrm{H}(2 ; 56)=12,011 ; \mathrm{p}=0,0025$

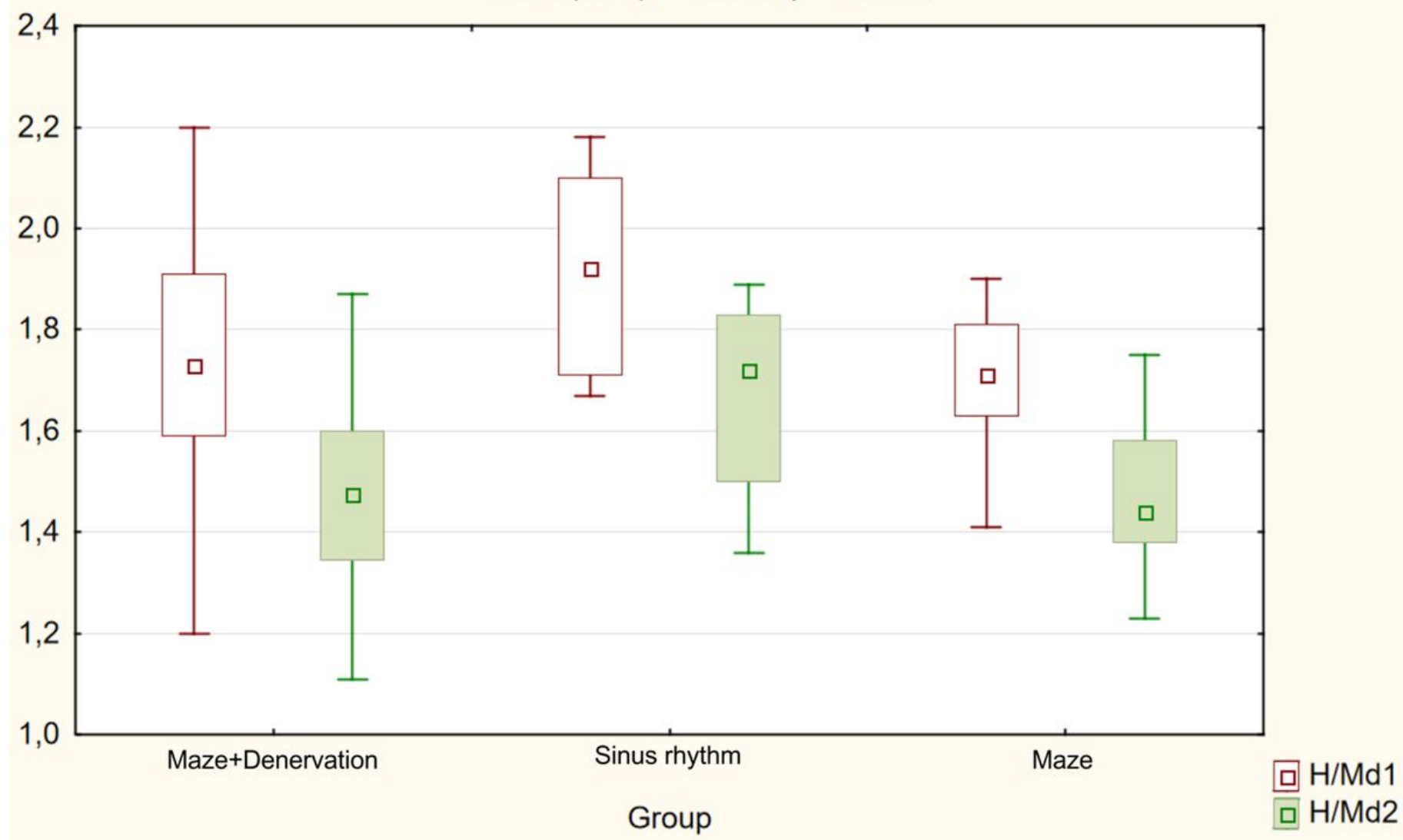

Figure 3

Heart-to-mediastinum ratio in the late phase of the study in the group with SR and AF 
RP washout rate before (WR1) and after (WR2) surgery

WR1: $F(2 ; 51)=1,3134 ; p=0,2778$;

$\mathrm{KW}-\mathrm{H}(2 ; 54)=2,5549 ; \mathrm{p}=0,2787$

WR2: $F(2 ; 53)=0,2979 ; p=0,7436$;

$\mathrm{KW}-\mathrm{H}(2 ; 56)=0,3892 ; \mathrm{p}=0,8231$

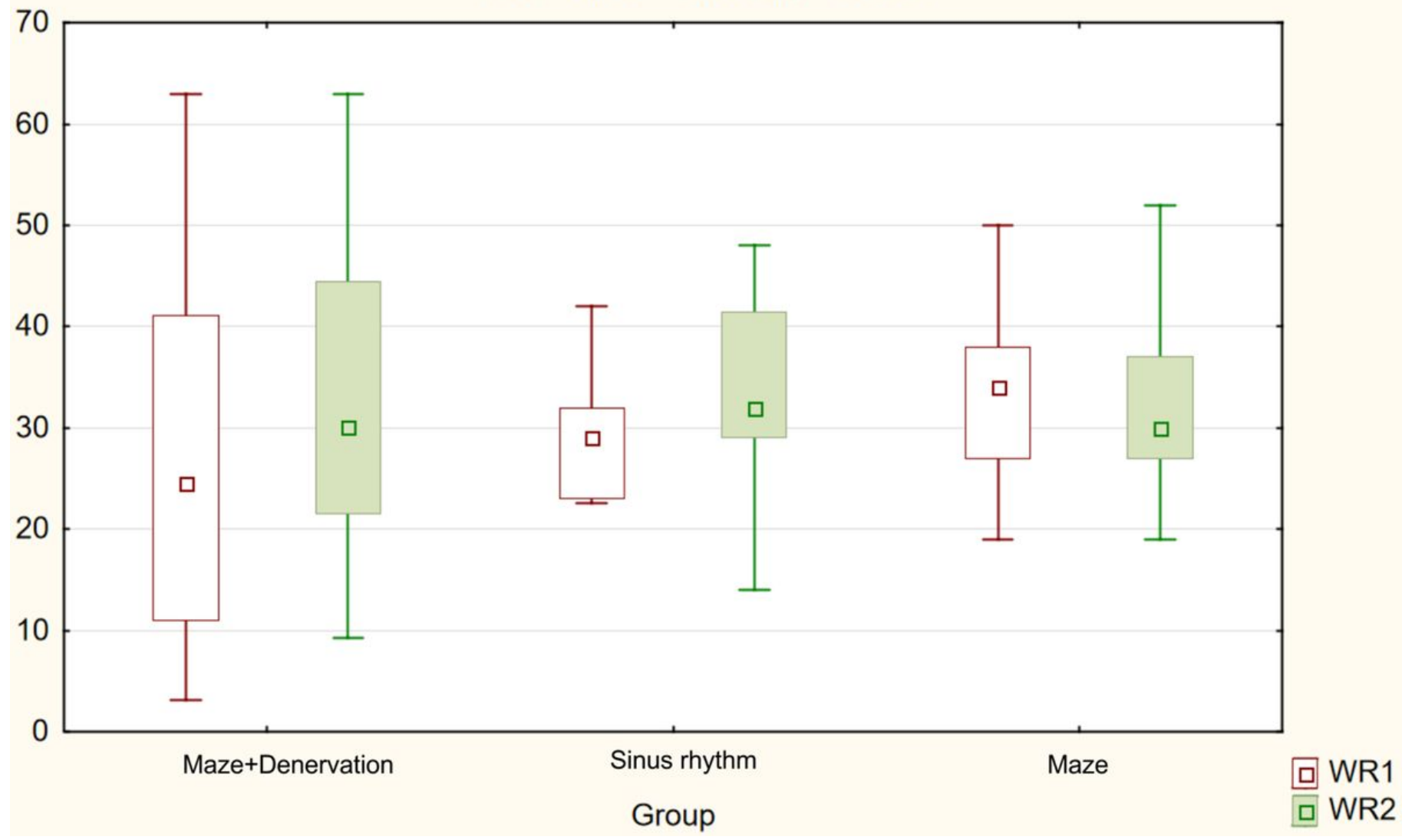

Figure 4

RP washout rate 
RP utake defect before (SRS1) and after (SRS2) surgery

SRS1: $F(2 ; 51)=0,658 ; p=0,5222$;

$\mathrm{KW}-\mathrm{H}(2 ; 54)=0,1822 ; \mathrm{p}=0,9129$

SRS2: $F(2 ; 53)=17,0046 ; p=0,00000$;

$\mathrm{KW}-\mathrm{H}(2 ; 56)=22,621 ; \mathrm{p}=0,00001$

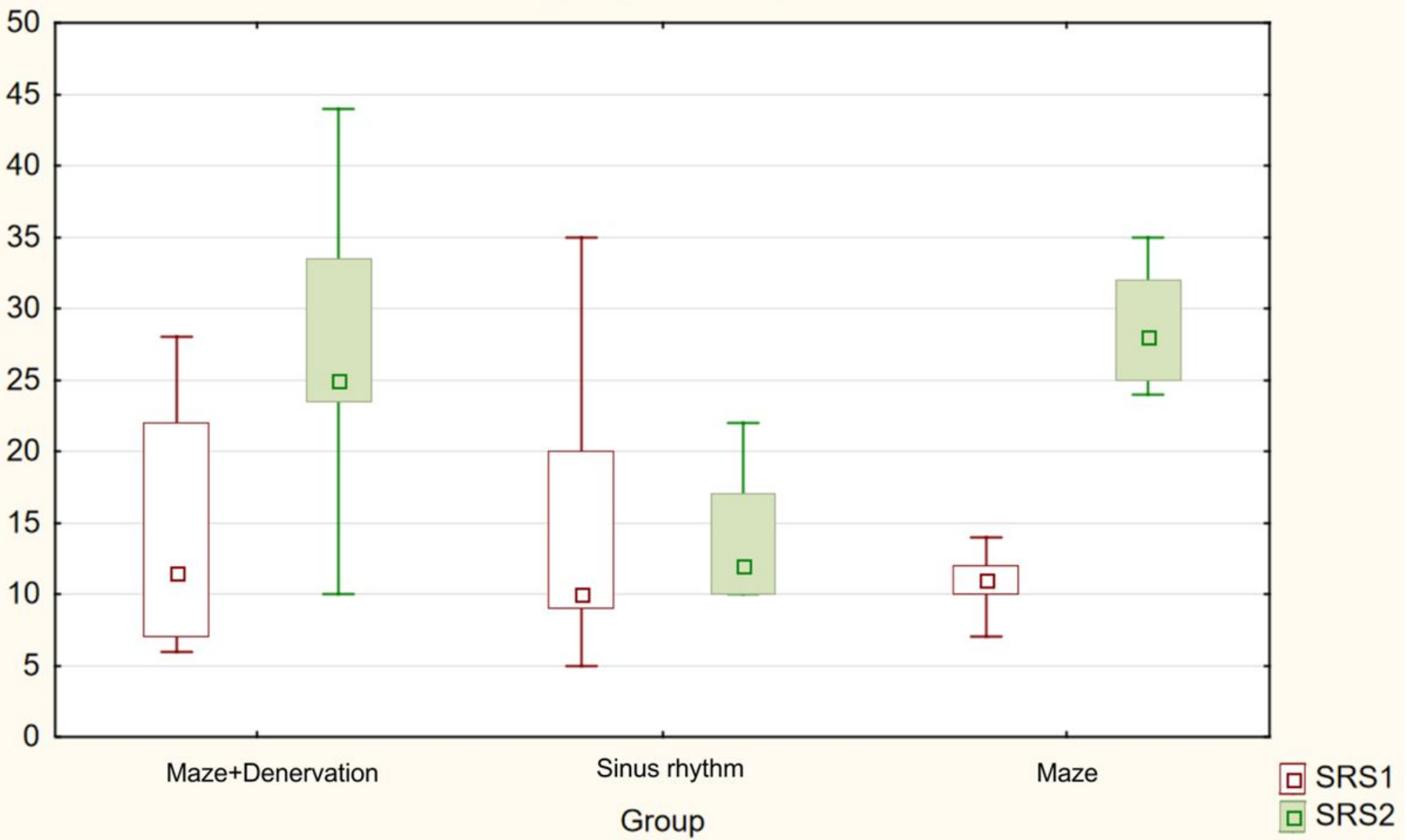

Figure 5

RP uptake defect

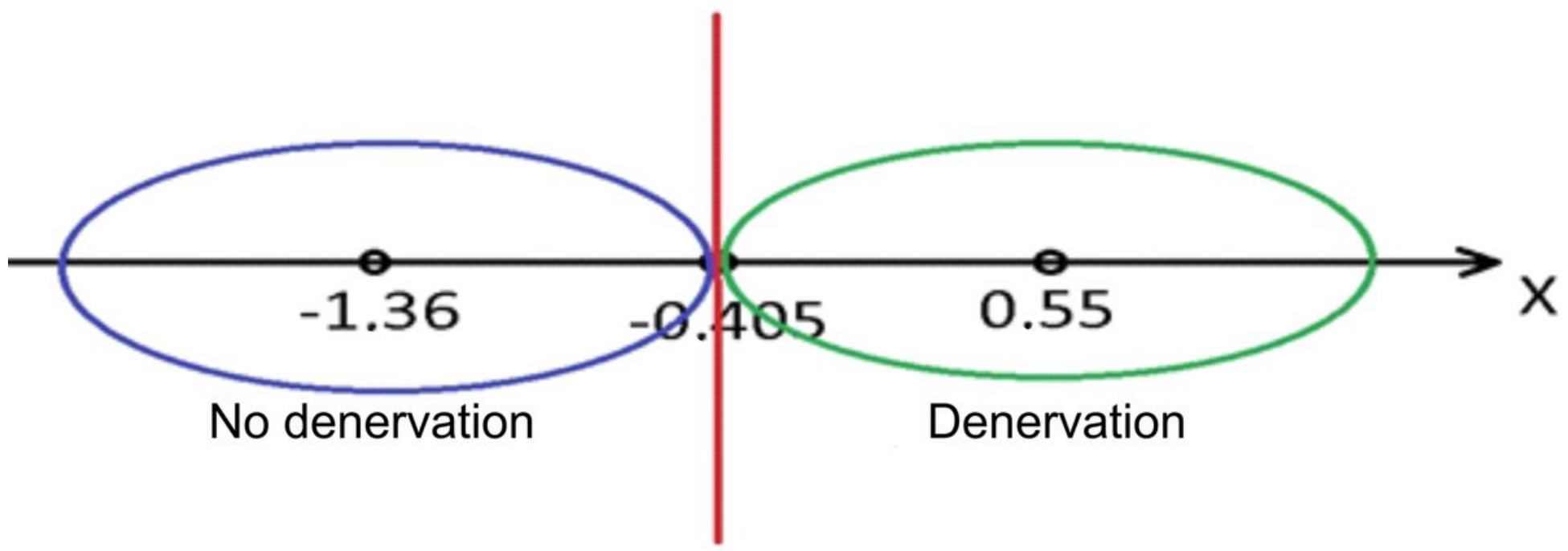

Figure 6

Model of quality assessment of RF denervation based on discriminant analysis 